\title{
ESTUDIO DE LAS DIFERENTES TÉCNICAS DE PRODUCCIÓN EN EL OLIVAR. SITUACIÓN ACTUAL Y PERPECTIVAS
}

\author{
POR \\ MARÍA JOSÉ CUESTA AGUILAR \\ $\mathrm{Y}$ \\ ANTONIO DELGADO CUENCA
}

\section{Consideraciones previas}

En el presente trabajo tratamos de aproximarnos al estudio de las consecuencias de la práctica de los sistemas de labranza tanto tradicionales como los más novedosos (cultivo sin labranza en sus diversas versiones), sobre la variación en la erosión hídrica en las tierras de olivar de la Cuenca del río Guadajoz.

Los efectos negativos que está sufriendo el medio ambiente son producidos, en un alto porcentaje, por el mal uso en las técnicas de manejo agrícola por parte de los agricultores.

El suelo agrícola está sufriendo impactos por erosión cada día más importantes. Se está produciendo un progresivo emprobrecimiento de sus características minerales y orgánicas, de su microfauna y de su fertilidad natural.

María José Cuesta Aguilar. Departamento Análisis Geográfico Regional y Geografía Física. Universidad de Granada.

Antonio Delgado Cuenca. Aceites Simera. Atarfe (Granada). 
La degradación aparece cuando el índice de erosión es mayor que el de la regeneración del suelo. La intensidad de las precipitaciones son fundamentalmente las causantes de la erosión. Su nivel de erosividad es muy alto.

Todos estos factores señalados (erosión del suelo, escorrentía, pérdida de agua), los vamos a encontrar en las zonas de olivar del área de estudio.

\section{Localización del área de estudio}

Aproximadamente, el $60 \%$ del olivar español se encuentra en Andalucía. Esta región es la primera productora de aceite de oliva. Se localiza en comarcas con pluviometría generalmente escasa, de distribución irregular (veranos secos) y con una evaporación potencial elevada.

Dentro de Andalucía hay dos provincias olivareras por excelencia: Jaén en primer lugar y Córdoba que le sigue. La cuenca del Guadajoz queda enmarcada dentro de estas dos provincias, ocupando el área Sureste de Córdoba y el extremo Suroccidental de Jaén. Sevilla, Málaga y Granada están en un punto medio en cuanto a producción del olivar y en Huelva, Cádiz y Almería, su importancia es mucho menor.

En la parte de la provincia de Jaén que entra en nuestra zona de estudio se incluyen dos comarcas (figura 1): Sierra Sur, donde se encuentran, entre otras, las poblaciones de Fuensanta de Martos, Valdepeñas de Jaén, Castillo de Locubín y Alcalá La Real. Tiene un 37 \% de olivar respecto al total provincial. El mismo porcentaje posee en cuanto a intensidad de cultivo, con carácter muy concentrado. $\mathrm{Su}$ productividad media es del 50,1\%. La relación suelo/clima es óptima en un $39 \%$ y existe muy poco olivar marginal.

La segunda comarca de la zona es la de Jaén-Martos, dentro de la cual se encuentra el término de Alcaudete. Su porcentaje de olivar sobre el total provincial es del $63 \%$. Algo inferior es la intensidad de cultivo, es decir una proporción muy alta. La productividad media es del $44 \%$, la relación suelo/clima es óptima en mayor porcentaje que en la comarca anterior, ya que llega al $56 \%$, y el porcentaje en olivar marginal es mínimo. 


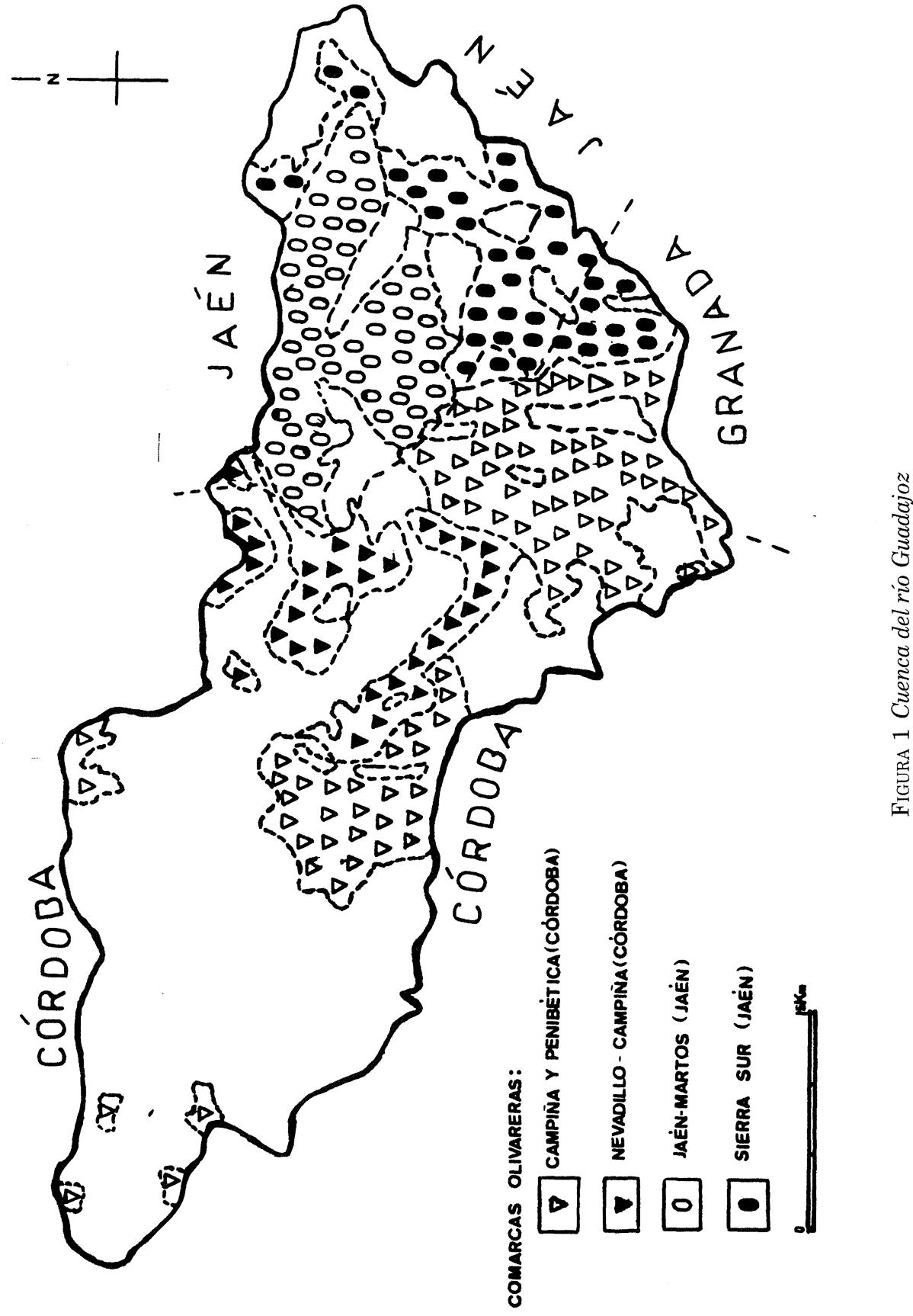


Respecto a la provincia de Córdoba, hay poblaciones incluidas en la Cuenca del Guadajoz que se enmarcan en el ámbito de otras dos comarcas: Nevadillo-Campiña, donde aparecen los términos de Valenzuela, Baena y Luque, con un porcentaje de olivar sobre el total provincial del $49 \%$. El cultivo es muy concentrado en un $32 \%$. La productividad media es del $61 \%$. La calificación de la relación suelo y clima es del $20 \%$ y no existe olivar marginal.

El resto de la cuenca ocupa la comarca denominada Campiña-Penibética. En esta última, el porcentaje provincial baja un poco respecto al anterior, ya que es del $41 \%$. El cultivo es muy concentrado en un 25,5\%. La productividad media es del 59,5\%. La calificación óptima entre suelo y clima es muy alta, del $54 \%$, y tampoco existe olivar marginal.

\section{La erosión hidrica}

El flujo temporal del agua en movimiento que afecta tanto a superficies inclinadas como a áreas con pendientes, tiene poder para arrancar, transportar y sedimentar partículas en gran cantidad.

Cuando la escorrentía superficial se produce, después de un aporte masivo de agua que consiga saturar el suelo, la erosión por arroyada areolar que sólo cumplía una función de lavado por medio de hilillos de agua, muy abundantes e inestables (rill), arrastra los elementos que encuentra de una manera difusa (rill wash o arroyada difusa en hilos). Cuando estos hilos de agua aumentan su caudal, su velocidad y su turbulencia, la erosión se hace concentrada, la incisión es mayor y aparecen las cárcavas.

Si la cárcava es profunda no desaparece en la época de inactividad hídrica y cuando de nuevo tengan lugar fuertes precipitaciones se reanudará el flujo de agua. El proceso de excavación reaparece y la concentración del agua de escorrentía es mayor, pudiendo llegar incluso a incidir en el sustrato rocoso.

Si la zona en concreto está desprovista de vegetación, quedará más afectada por esta acción. Igual ocurre si predominan los materiales margosos y arcillosos.

$$
-440-
$$


La erosión provocada por esta acción geomorfológica está dando lugar a pérdidas importantes de suelo y de agua aprovechable para el cultivo de olivar, sobre todo en la comarca de la Subbética.

Con el laboreo tradicional en el olivar, el exceso de labranza favorecía la erosión, pero con el cambio de esta técnica al no-laboreo, técnica adoptada posteriormente tampoco se resuelve el problema, más bien se acrecienta ya que la compactación de la capa superficial de la tierra al rulearla provoca el fuerte aumento de la escorrentía al descender drásticamente la infiltración, y aumentar por tanto la pérdida de agua. El problema es mayor cuando aparecen las cárcavas que, al no ser borradas, se van afianzando, pasando con los años a convertirse en irreversibles. En muchos casos, llegan a ser auténticos canales de desagüe que suponen una gran pérdida no sólo ambiental, sino también, a no muy largo plazo, productiva.

\section{Los sistemas tradicionales}

En la actualidad, el sistema de manejo más empleado en Andalucía en el cultivo del olivar es el laboreo mecánico. Los aperos más usuales son el cultivador, la grada de discos, el vibrocultivador y la «rastra» o grada de púas. La desventaja básica que causan en el olivar es la destrucción de las raíces.

Las variedades más comunes de olivar en la zona de estudio sontres:

«Picual»: Es la que tiene más ventajas por su mayor producción y con la mayor regularidad. El contenido de aceite en aceituna es del $22 \%$. Responde bien a la recolección (vareo) y a la poda. Sin embargo es exigente en suelos y resiste poco la sequía. En la zona de Luque, Baena y Fuente-Tójar es dominante.

«Hojiblanca»: Es menos exigente respecto a los factores ambientales pero produce menos y su contenido graso es sólo del $18 \%$. Se encuentra, en general, en el sur de Córdoba sin poderse determinar con exactitud áreas concretas.

«Picudo»: Es la variedad de más rendimiento (26\%) y calidad, pero no produce mucho y necesita de cuidados en la poda, el vareo y el tratamiento de las plagas. Es la dominante en la zona de estudio, 
ya que se encuentra en la línea que une Almedinilla, Fuente-Tójar, Priego, Carcabuey, Luque, Baena y Castro del Río en Córdoba y Alcaudete en Jaén.

Los principales inconvenientes del sistema de laboreo en olivar tradicional es la intensificación hasta la exageración en el empleo de la grada de discos en cuanto a la labor. Respecto a la poda se llegó al uso y abuso de la motosierra y en el abonado era frecuente entre los agricultores aplicar el producto «de moda», sin asesoramientos técnicos. El «labrar mucho» por el «labrar bien» ha provocado la pérdida de fertilidad del suelo, sobre todo a los olivares situados en laderas, los cuales están siendo profundamente afectados por la erosión. Desde un punto de vista ambiental distinguimos factores físicos y técnicas de cultivo.

Factores físicos. Las condiciones óptimas para el cultivo del olivar son las del clima mediterráneo, con una temperatura media anual de 15 a $20^{\circ}$. Se puede alcanzar una máxima de $40^{\circ}$ y una mínima de $-8^{\circ}$, aunque estas temperaturas no deben ser frecuentes ni continuas, ya que la resistencia del olivo depende de la relación duración/intensidad. El olivo necesita de una mínima amplitud térmica, ya que el frío invernal es necesario para la diferenciación entre las yemas productivas y las vegetativas.

En nuestra zona de estudio, el clima es bastante apto, sobre todo para el desarrollo vegetativo, pero irregular en cuanto a su producción.

Las características térmicas en la cuenca, están en torno a los $15^{\circ}$ de media anual, las máximas superan los $35^{\circ}$ y las mínimas oscilan alrededor de los $3^{\circ}$, es decir no rebasan los límites del espectro térmico óptimo.

La pluviometría oscila entre los 400 y los 1.000 litros anuales en la zona. Los límites pluviométricos del olivo están entre los 200 y los 1.000 litros. Para obtener una buena producción, el régimen de humedad debe ser relativamente beneficioso. En parcelas experimentales de la provincia de Jaén se ha podido comprobar la relación directa entre año lluvioso y buena producción.

Las grandes oscilaciones climáticas interanuales en este área, que aparecen con cierta regularidad hacen que la producción anual varíe considerablemente. 
Respecto a los suelos, el olivo necesita que sean francos, tanto calizos como silíceos. Los suelos óptimos son los que se encuentran en el piedemonte mioceno del Subbético: las rendsinas de humus mull, los suelos rojos mediterráneos y la tierra parda caliza. Los «bujeos», característicos de la Campiña, son suelos arcillosos y por su comportamiento son más indicados para los cultivos intensivos de secano (trigo, girasol) que para el olivar, ya que los polígonos que forman al resecarse por la falta de lluvia destruirían sus raíces.

La altitud más adecuada se circunscribe a una banda situada en tre los 200 y los $800 \mathrm{~m}$, y la mayor parte de la cuenca del Guadajoz está por debajo de los $800 \mathrm{~m}$, salvo puntos muy concretos.

Tradicionalmente se ha plantado el olivar en terrenos con alguna pendiente, la cual ha impedido la siembra de otro cultivo. Las zonas más bajas, por su mal drenaje o por el mayor peligro de heladas se han cultivado con otro tipo de aprovechamientos.

Técnicas de cultivo. Los marcos de plantación más frecuentes estaban entre $10 \times 10$ y $12 \times 12 \mathrm{~m}$ en cuadros, o al tresbolillo; la densidad media era de 80 a 100 árboles por hectárea. Las estacas se empleaban para la multiplicación vegetativa porque enraizaban fácilmente. Actualmente, se cultivan en vivero y desde aquí se trasplantan. La densidad está en torno a los 200 árboles por hectárea, con un marco real de $7 \times 7 \mathrm{~m}$.

Respecto a las labores, hay que hablar en primer lugar de la llamada «alzar», que consiste en hacer huecos en el suelo para retener el agua y destruir las malas hierbas. Se hace en otoño, con el empleo de la reja. La labor de «abrir» (procedimiento análogo al anterior) se realiza con escarificador o grada de discos. Del otoño a la primavera, se dan como mínimo dos pases de reja profunda.

Cuando terminan las lluvias de primavera aparecen las labores de gradeo que duran todo el verano. En el primer pase se trata de arrancar las malas hierbas que han crecido en primavera, lo cual supone la pérdida de la materia orgánica que tendría el suelo. Después se suceden los pases de grada de púas o escarificador en julio y agosto, y parte también de junio y septiembre. Con ello se tapan los estomas y se reduce la evapotranspiración al «dar polvo». 
La arroyada laminar es predominante cuando el olivar se cultiva de esta forma tradicional. Las cárcavas se corrigen al labrar, no se les facilita el camino para que se instalen definitivamente como ocurrirá en la técnica de no-laboreo donde la arroyada se concentrará a través de ellas.

Cuando la longitud y la superficie de una cuenca hidrológica son lo suficientemente amplias, la erosión difusa o laminar propia de la cuenca sufre un cambio cualitativo, a favor de la erosión concentrada, sobre todo en las cotas más bajas de las vertientes. La erosibilidad del suelo se reduce y ocurre al contrario con la velocidad del agua que aumenta al crecer la escorrentía superficial.

\section{El cultivo sin labranza}

El no-laboreo es un sistema de cultivo donde se suprimen las labores controlándose las malas hierbas a base de herbicidas. El resto de las operaciones (tratamientos, poda, etc.) se sigue realizando de la misma manera que en el olivar tradicional. Este sistema se debe iniciar al final del verano o principios del otoño dejando el terreno liso y a ser posible ruleado, aplicando entonces herbicida de preemergencia que es normalmente Simazina y siempre antes de que nazca la hierba.

La realización de las obras de defensa es fundamental para ęvitar la erosión del suelo y la pérdida de agua. Éstas serían: a) construcción de pozas; b) surcos o caballones; c) albarradas y gaviones; d) corta-cárcavas: zanjas perpendiculares a la línea de máxima pendiente, relleno de poda, construcción de caminos compactados...; e) aterrazado; f) ahoyado (en el olivar plantado en tresbolillo).

En USA comenzó a practicarse esta técnica con el cultivo de agrios en 1913. Posteriormente se ha ido extendiendo a otros cultivos, herbáceos también, como el maíz, por ejemplo. Otros países citrícolas han aplicado también esta técnica, entre ellos destacan Israel, Japón y España.

En España comenzó el no-laboreo en 1962, en un olivar marginal de la zona de Cambil, provincia de Jaén. Pero será en los años setenta cuando se generaliza el cultivo del olivar sin labrar a la vez que el empleo de herbicidas. En el no-laboreo se pueden distinguir tipos: 
- El no-laboreo con suelo desnudo, donde se suprimen totalmente las labores. El suelo se alisa y se controlan las malas hierbas con simazina en otoño, y glifosato u otro herbicida en primavera.

- Sistemas mixtos o semilaboreo: a) sistema de «Ruedos permanentes», donde se apisona alrededor del $40 \%$ del terreno proyectado bajo la copa. Es aquí donde se aplica el herbicida. El resto se labra de forma convencional. b) Laboreo mínimo en franjas, en el que alternan franjas labradas una sola vez al año en el sentido de las curvas de nivel, y franjas sin labrar bajo la copa, aplicando aquí herbicida, y por tanto con suelo alisado.

Actualmente, el laboreo sigue siendo el modo más empleado de cultivar el olivar. Le sigue en importancia el semilaboreo o de «ruedos permanentes» que a veces es de franjas. Habría que distinguir una serie de ventajas e inconvenientes desde el punto de vista socioeconómico y desde el medioambiental.

Siguiendo a Pastor (1990), se puede decir que desde la perspectiva socio-económica el no-laboreo reporta una serie de ventajas:

- Aumento medio de la cosecha hasta un $30 \%$.

- Reducción de las heladas primaverales, por la mayor conductividad y difusividad térmica del terreno.

- Menores costos de producción por: reducción del consumo de energía (gasóleo); de la inversión en maquinaria; de los costes en la recolección; también aumenta el número de días útiles de trabajo en el campo durante el invierno al presentarse el suelo más compactado, ya que es posible el tránsito de personas y vehículos una vez finalizado el período de precipitaciones.

- En olivares con goteo, es el sistema más cómodo de utilizar.

Desde la perspectiva ambiental, la planta obtiene también una serie de ventajas:

- Mejora la estructura del suelo al desaparecer la suela de labor, pero podríamos decir que esto es sólo una mejora relativa, ya que ese problema se «traslada» a la superficie por la fuerte 
compactación que sufre la capa superior al ser apisonada por el rulo.

- Se reducen las pérdidas de suelo por erosión al aumentar la estabilidad estructural y la resistencia de las partículas al transporte.

- Aumenta su sistema radicular, al aprovechar mejor la capa superior de tierra que es la más fértil. También recoge las lluvias escasas, lo que influye en su mayor crecimiento vegetativo.

- A veces se ha justificado el laboreo porque destruye la vegetación adventicia que compite por el agua del terreno. Los herbicidas empleados en régimen de cultivo sin laboreo lo sustituyen y lo superan en economía, eficacia y control de las malas hierbas.

- Se reducen las pérdidas de agua por evaporación, aunque sólo en precipitaciones de escasa cuantía, ya que en el caso de una lluvia fuerte la pérdida de agua por escorrentía es mayor. Además de nuevo la compactación del suelo provoca también que la infiltración sea más baja.

Desde la visión de la rentabilidad económica también pueden citarse una serie de inconvenientes:

- La mayor actividad del árbol al producir más, provocará que el déficit hídrico aparezca antes que en el olivo labrado, lo que influirá al final en la peor calidad del fruto, por su menor tamaño, su rendimiento graso más bajo y en consecuencia un menor valor comercial.

- Cuando la incorporación del abono es imposible por la compactación del suelo, y las cárcavas impiden el paso de la maquinaria, el agricultor suele abandonar esta técnica.

Los inconvenientes que se pueden citar teniendo en cuenta su repercusión en el medio ambiente son mayoritarios:

- Disminución de la tasa de infiltración por el impacto de las gotas de lluvia en el suelo y por el tránsito de la maquinaria y los 
operarios fundamentales, que causan la compactación del suelo y la formación de costras que favorecen la escorrentía concentrada, factor muy a tener en cuenta de cara a la predicción de riesgos por inundaciones. Este encostramiento se formaría normalmente debido a las características del suelo arcilloso de la zona de la cuenca del río Guadajoz. Se suma también el efecto del ruleo a la compactación del suelo, con lo que el problema se agrava al practicar esta técnica. Esta escorrentía facilita la erosión, transporta los coloides y la materia orgánica del suelo, junto a los herbicidas, fundamentales en el no-laboreo para el control de las malas hierbas.

- En ciertos estudios se ha demostrado que la simazina, que es el herbicida más empleado para controlar las malas hierbas en nolaboreo, puede dar lugar a fitotoxicidad en árboles jóvenes, en suelos muy calizos y erosionados, y en terrenos altamente erosionables donde los arrastres de tierra puedan sedimentar en otras parcelas con cultivos sensibles a este producto. La simazina es un herbicida residual (que es aquel que queda en el suelo si bien su acción se prolonga durante las doce primeras semanas de su aplicación), de escaso grado de percolación, por lo que se mantiene en niveles edáficos superficiales y no se ve especialmente afectado por la luz. Actúa eficazmente sobre la mayor parte de las especies herbáceas anuales de ciclo invierno-primavera, o primavera-verano, pero es poco activo sobre hierbas perennes.

- Son indudables las múltiples ventajas que presenta el uso de los herbicidas para el agricultor, ya que lo exime de la tediosa labor que supone la eliminación manual de la vegetación espontánea, y además en períodos que por condiciones meteorológicas adversas no sería posible el control manual de este tipo de vegetación.

Frente a las ventajas mencionadas, el uso incorrecto de los herbicidas puede presentar los siguientes inconvenientes:

- Desarrollo de adaptaciones de estas plantas frente a las dosis habituales. Hay que tener en cuenta el concepto de dosis cuando tratamos de aplicación de los herbicidas. 
- Aparición de fitotoxicidad en el olivo. Ésta suele ser la consecuencia del empleo de dosis inadecuadas y de fallos en la aplicación del herbicida, así como del uso impropio de éstos.

- Residuos de herbicidas sobre las cosechas y los productos derivados de ellas, con la consecuente declaración de no aptos para el consumo humano.

- Residuos de herbicidas que provocarán contaminación fluvial debido a la evacuación de éstos a través de las vertientes, así como posible contaminación de acuíferos subterráneos por percolación de los sucesivos horizontes edáficos.

- Toxicidades directas sobre el hombre, animales domésticos y fauna silvestre.

- Posible inversión de flora.

Por último hay que señalar que el uso correcto del herbicida no implica un uso racional ni óptimo sino adaptar reglas y conocimientos que deben ser dominados por los diferentes sectores: fabricante, olivarero y Administración.

\section{Los nuevos métodos de producción}

No vamos a limitarnos sólo a describir una técnica y los problemas que conlleva. Queremos hacer una crítica constructiva, por lo que proponemos una solución al problema, que hemos conocido recientemente y que podemos considerar una experiencia pionera en el cultivo del olivar. Se trata de cultivo sin labranza, realizado en una finca con una pendiente media del $20 \%$. Aporta una novedad que consiste en dejar una cobertura vegetal entre las calles. Esta cubierta está formada por la vegetación que crece de forma espontánea entre las calles de la plantación, la cual se desbroza antes de que entre en competición con el olivo por el consumo de agua. La proyección de la copa del árbol sí se mantiene con suelo desnudo aplicando ruleo y herbicida.

Este sistema es una poderosa alternativa, ya que no tiene ningún inconveniente porque reduce al mínimo el coste de la maquinaria

$$
-448-
$$


(sólo es necesario una desbrozadora, una trituradora y un vibrador para la recolección), de la poda y de la mano de obra. Además el coste de los herbicidas es mínimo, y el del abono es prácticamente nulo, ya que la misma cubierta vegetal se utiliza como abonado orgánico. Los resultados en la producción son óptimos.

Respeta al máximo el medio ambiente y no crea los problemas que encontrábamos en los sistemas descritos anteriormente: erosión, cárcavas, escorrentía... El mantenimiento de la cubierta retiene y mantiene el agua, la pérdida por escorrentía desaparece. Las cárcavas no llegan a crearse y no existen pérdidas de suelo de ningún tipo. Además, el ramón triturado se esparce y distribuye por el suelo, sirviendo de matriz para la reproducción de las hierbas en aquellas zonas donde la erosión fue más grave. Podríamos hablar de agricultura ecológica, ya que se acerca al máximo a lo que sería una producción natural.

Este sistema de cultivo se ha llevado a la práctica en la finca «Dehesas del Tobazo», en el término municipal de Alcaudete (Jaén). A ella hacíamos referencia al comienzo de nuestro artículo.

\section{Conclusiones}

Está demostrado que el no-laboreo es una técnica con la que se han conseguido importantes avances desde el punto de vista económico, de cara al agricultor. Pero éste no lo practica en su totalidad o sea como los técnicos lo aconsejan, es decir llevando a cabo las medidas anti-erosión para la contención de tierra y agua.

La falta de información del agricultor, la negligencia o la búsqueda exclusivamente de la rentabilidad económica inmediata hacen que el problema del cambio cualitativo en el sistema hídrico y su componente respecto al riesgo natural que conlleva sea cada vez mayor.

No compartimos la opinión de López Ontiveros, A. (1980), cuando dice: «La labranza es un problema económico antes que técnico, si se dispone de dinero y el árbol va a responder, el agricultor gastará dinero; si no, con mucha razón, no gastará un duro.» 
La realización de obras de defensa para limitar la escorrentía y disminuir así el impacto del agua, el encostramiento superficial, la continuidad del proceso a lo largo de toda la vertiente y la velocidad del agua es la única solución viable en los olivares con estos problemas. El problema (desde la perspectiva de la rentabilidad), sería que la producción es algo inferior a la producida bajo régimen de no-laboreo, y su coste económico sería mayor por lo que el cambio hacia un nuevo sistema -mantenimiento en no-laboreo del ruedo del olivo y cubierta vegetal en el resto del terreno-, es a nuestro juicio la solución óptima, ya que es el único sistema donde no encontramos inconvenientes de ningún tipo.

No se puede quitar importancia al problema de la pérdida de suelos por erosión. Las ventajas económicas del sistema no pueden pesar más que sus aspectos negativos. Sin embargo, a partir de experimentos realizados con simuladores de laboratorio, o en parcelas pequeñas donde la longitud de la cuenca «natural» se acorta, los resultados serán más ventajosos que cuando se estudia una parcela completa en la que se están midiendo parámetros reales donde, de forma natural, se han tenido en cuenta todos los factores, sin ponderaciones ni aproximaciones.

Por otro lado, los modelos que se adecúan a unas determinadas y concretas características, no se pueden generalizar a otros puntos geográficos, ya que no son extrapolables. Los resultados estarían simplificados y falseados.

Hay que calificar de indeseable y nada recomendable, desde el punto de vista ambiental, el cultivo del olivar con este sistema mantenido hasta ahora. Está admitido que, por lo general, en suelos con pendientes superiores al $5 \%$ (la mayor parte de la zona que nos ocupa), los inconvenientes de esta técnica se intensifican, y, ante el reiterado incumplimiento de las medidas anti-erosión, el problema sigue creciendo.

Con este sistema de cultivo se está produciendo un aceleramiento inducido de la erosión, que implica un ritmo excesivamente rápido de pérdida de suelos productivos $\mathrm{y}$, tal como hemos podido comprobar en algunos puntos, su anulación por reducción al mínimo de su espesor. 


\section{BIBLIOGRAFÍA}

INIA (1988). Las comarcas olivareras andaluzas. Madrid, MAPA.

López Ontiveros, A. (1980). ¿Qué pasa con el olivar? Granada, IDR.

Muñoz Jiménez, J. (1992). Geomorfología General. Espacio y Sociedad. 4. Madrid, Síntesis.

Ortega Alba, F. (1975). El sur de Córdoba. Estudio de Geografía Ágraria. 2 tomos. Córdoba: Public. del Monte de Piedad y Caja de Ahorros de Córdoba.

Pastor Muñoz-Cobo, M. (1990). El no laboreo y otros sistemas de laboreo reducido en el cultivo del olivar. Sevilla, DGIEA.

Resumen: El uso inadecuado e incompleto de las técnicas de manejo de cultivo del olivar en la cuencia del río Guadajoz venía provocando una serie de problemas medioambientales que se han visto agravados a consecuencia de los cambios en la erosión hídrica que se están produciendo en el sistema de cultivo denominado no-laboreo, por el aumento de la escorrentía y el descenso de la infiltración.

Palabras Claves: Olivar, erosión hídrica, no laboreo, Guadajoz.

RESUMÉ. L'utilisation inapropiée et incompléte des techniques du culture de l'olivier dans le bassin du Guadajoz a provoqué des nombreaux problèmes pour l'environment. Les problèmes ont été aggravés a cause des changements dans l'érosion hydrique, produits dans le systéme de culture nomné non labouré, du á la diminution et l'augmentation dui ruissellenent.

Mots CLÉ: Olivier, érosion hydrique, non labouré, Guadajoz.

AbSTRACT. The inadequate and incomplete use of handling technique for olive grove cultivate at Guadajoz river basin has caused a series of environmental which has been increased because of the changes in water erosion which is being produced in the cultivate system called "without exploitation", because of the infiltration decrease and the slippery increase.

KEY WORDS: Olive, water erosion, «without exploitation», Guadajoz. 